\title{
Postprandial Symptoms in Patients With Functional Dyspepsia and Irritable Bowel Syndrome: Relations to Ultrasound Measurements and Psychological Factors
}

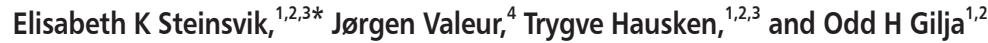 \\ ${ }^{1}$ Department of Clinical Medicine, University of Bergen, Bergen, Norway; ${ }^{2}$ National Center for Gastrointestinal Ultrasound and ${ }^{3}$ National Center \\ for Functional Gastrointestinal Disorders, Department of Medicine, Haukeland University Hospital, Bergen, Norway; and ${ }^{4}$ Unger-Vetlesen \\ Institute, Lovisenberg Diaconal Hospital, Oslo, Norway
}

\section{Background/Aims}

Dyspeptic symptoms are common in patients with functional gastrointestinal (GI) disorders, and may be related to visceral hypersensitivity. We aim to explore the relation between visceral hypersensitivity by using an ultrasonographic meal test and questionnaires in patients with irritable bowel syndrome (IBS) and/or functional dyspepsia (FD).

\section{Methods}

Patients (FD, $n=94 ;$ IBS, $n=88$; IBS $+F D, n=66$, healthy controls $[H C], n=30$ ) were recruited consecutively and examined with ultrasound of the proximal and distal stomach after drinking $500 \mathrm{~mL}$ of a low caloric meat soup, and scored dyspeptic symptoms on a visual analogue scale (0-100 mm) before and after the meal. Psychological symptoms were assessed by Visceral Sensitivity Index (GI specific anxiety, $n=58$ ), and Eysenck's Personality Questionnaire-neuroticism (EPQ- $N, n=203$ ).

\section{Results}

Patients with IBS and/or FD reported higher levels of nausea, upper GI discomfort, and epigastric pain both before and after a liquid meal compared to HC $(P<0.001)$, and had a larger antral area in a fasting state, compared to HC. We found impaired accommodation in $33 \%$ of the patients with FD, however ultrasound measurements and symptom severity did not correlate. Symptoms of epigastric pain, fullness and upper GI discomfort positively correlated to Visceral Sensitivity Index and EPQ-N in a fasting state, but not postprandially.

\section{Conclusions}

Nausea, upper GI discomfort, and epigastric pain was common in patients with IBS and FD. Both patient groups had enlarged antral area in a fasting state compared to HC. Discomfort and pain were associated to Gl specific anxiety and neuroticism in a fasting state.

(J Neurogastroenterol Motil 2020;26:96-105)

Key Words

Anxiety; Gastrointestinal diseases; Nausea

Received: March 31, 2019 Revised: August 13, 2019 Accepted: September 20, 2019

(5) This is an Open Access article distributed under the terms of the Creative Commons Attribution Non-Commercial License (http://creativecommons. org/licenses/by-nc/4.0) which permits unrestricted non-commercial use, distribution, and reproduction in any medium, provided the original work is properly cited.

*Correspondence: Elisabeth K Steinsvik, MD

Medical department, Haukeland University Hospital, Pb 1400, 5021 Bergen, Norway Tel: +47-55970508, Fax: +47-55972950, E-mail: Elisabeth.Steinsvik@uib.no 


\section{Introduction}

Functional gastrointestinal disorders (FGIDs) such as functional dyspepsia (FD) and irritable bowel syndrome (IBS) are common conditions affecting daily life and activities of many patients. The pathophysiology of FGIDs is complex and not fully understood, involving disturbances of the brain-gut-axis, low-grade inflammation, gastric and intestinal dysmotility and visceral hypersensitivity, as well as changes of the microbiota. ${ }^{1-3}$ In FD the main symptoms are early satiation, epigastric pain, postprandial fullness, and epigastric burning. ${ }^{4-6}$ IBS is characterized by recurrent abdominal pain associated with stool changes, sometimes with abdominal bloating and distention. ${ }^{2}$

Dysmotility and hypersensitivity of the stomach are key features in $\mathrm{FD},{ }^{7}$ and has been extensively studied by many different modalities. Symptoms in FGIDs are often exacerbated by food, and meal tests are a common method of investigating postprandial symptoms. $^{8}$

In addition to the before mentioned mechanisms underlying the FGIDs, anxiety and depression are frequently reported in association with FGIDs, ${ }^{5,9}$ and may play an important role in modulating the sensory stimuli on the gut-brain axis. ${ }^{10}$ However, it is unclear if this relationship is an indication of causality or consequence. ${ }^{11}$

In this paper, we present results from the Ultrasound Meal Accommodation Test (UMAT) ${ }^{12}$ investigating the stomach by ultrasound, combined with a drink test, not only in FD patients, but also patients with IBS, and healthy controls (HC). The general findings from this cross-sectional study have been published in $2016 .{ }^{13} \mathrm{We}$ aim to investigate dyspeptic symptoms in patients with FGIDs, and explore whether the symptoms were associated with alterations of intragastric meal distribution assessed by the UMAT and psychological factors.

\section{Materials and Methods}

\section{Study Population}

All patients who had undergone the UMAT from 1999 to 2014 at Haukeland University Hospital, a tertiary University clinic in Bergen, Norway, were included ( $\mathrm{n}=509)$.

In the present study, we identified patients diagnosed with FD or IBS during the UMAT. Patients were diagnosed with FD or IBS according to Rome II and III, depending on time of inclusion. Following UMAT and a clinical evaluation, patients were given In- ternational Statistical Classification of Diseases and Related Health Problems (ICD-10) diagnoses of IBS and/or FD, following clinical routines. Patients with abdominal discomfort, pain, nausea, or dyspepsia clearly associated with depression or eating disorders were given the diagnosis "psychogenic abdominal pain" and treated as a separate group. Patients with signs and symptoms of gastroparesis, in particular patients with diabetes type 1 , were not diagnosed with FD.

HC ( $\mathrm{n}=30$; 20 women, 10 men) were recruited and examined during 2016 and 2018. Some were students in nursing and medical school; others were colleagues from our hospital. All signed written consent before enrollment. None of the controls had FD or IBS according to the Rome III or IV criteria, and they did not use any medications affecting the stomach. One participant had had an appendectomy, 2 had had ovarian surgery, and 1 had her uterus removed. They were all examined by the same protocol as the patients. One of the controls had an abnormal shape of her proximal stomach, and accordingly, these measurements were excluded from our analyses.

\section{Symptoms}

Visual analogue scales (VAS) has proven valuable to record nausea, and in some studies has proven superior to the Likert and Borg integral scales. Measuring change in the intensity of nausea after meal ingestion is recognized in diagnoses such as gastroparesis. ${ }^{14}$ In this project, patients reported their levels of epigastric pain, nausea, fullness/bloating, satiety and total discomfort on a VAS ranging from $0-100 \mathrm{~mm}$, at the fasting state and immediately after soup ingestion.

\section{The Ultrasound Meal Accommodation Test}

The UMAT is a clinical examination that combines accommodation testing, a drinking test and evaluation of gastric emptying. The procedure was previously described by Gilja et al. ${ }^{15}$ The patients were examined in a fasting state, seated, and leaning slightly backwards. Measurements of the area of the antrum was obtained by a curvlinear abdominal ultrasound probe (frequency $3.5 \mathrm{MHz}$ ), and the patient reported his/her symptoms using standardized symptom questionnaires (VAS) before drinking a $500 \mathrm{~mL}$ low-calorie soup (Toro klar kjøttsuppe; Rieber \& S $\varnothing n$ A/S, Bergen, Norway; contents: $1.8 \mathrm{~g}$ protein, $1.1 \mathrm{~g}$ carbohydrate, $0.9 \mathrm{~g}$ bovine fat, and $83.68 \mathrm{~kJ}$ ) during 4 minutes. Immediately after soup intake, the proximal stomach was measured in 2 standardized sections, and the antral area was measured. Symptom registration was repeated immediately after finishing the meal. Ultrasound measurements were 
repeated at 10 minutes and 20 minutes postprandially. By measuring the sagittal area, together with the greatest oblique diameter of the proximal stomach, it is possible to assess gastric accommodation by ultrasound. Only the patients who completed the $500 \mathrm{~mL}$ meal were included in the analysis of ultrasonographic measurements.

\section{Questionnaires}

As a measure of traits of neuroticism, we used a 12 item questionnaire (Eysenck's Personality Questionnaire-neuroticism [EPQ$\mathrm{N}$ ]; Revised, Short Form). ${ }^{16}$ The Visceral Sensitivity Index (VSI) is a questionnaire measuring gastrointestinal (GI) specific anxiety, and has proven useful in populations with FGIDs. ${ }^{17,18}$ The IBS Symptom Severity Score (IBS-SSS) was used to assess the symptom load of IBS symtptoms. ${ }^{19}$ VSI and IBS-SSS were introduced in 2013 and results were available for most patients examined during 2013 and 2014. EPQ-N has been used consistently from 1999. In some cases, patients did not answer all required fields, and the results from these questionnaires were excluded from analysis.

\section{Ethical Considerations}

The study was defined as a quality control project, and approved by the Data Protection Official at Haukeland University Hospital (2014/20478). Information about the study participants was stored anonymously on a secure server. The HC study was approved by the Regional Ethical Committee (REK 2014/222-20).

\section{Statistical Methods}

All analysis were done using IBM SPSS Statistics version 24 (IBM Corporation, Armonk, New York, USA), and figures were made using GraphPad Prism version 8 (GraphPad Software, San Diego, USA). Unless stated otherwise, data are presented as mean $\pm \mathrm{SD}$. We used the $\chi^{2}$ test and odds ratio to compare categorical variables. For the comparisons of continuous variables between independent groups, we used Students $t$ test, and for changes in symptoms before and after a meal, we used paired samples $t$ test. When comparing ultrasonographic measurements between the different patient groups and $\mathrm{HC}$, we used one-way ANOVA where the variances were similar (assessed by Levene's test), and Welch's test where the variances differed. Because of difference in sample sizes and sometimes variance between the groups, we used the Games-Howell post-hoc test. Associations were analyzed by Pearson's correlation, linear regression, and logistic regression. Results from logistic regression were reported as an odds ratio with $95 \%$ confidence interval. $P$-values $<0.05$ were considered statistically significant.

\section{Results}

\section{Study Population}

In the period from 1999-2014, 509 patients were assessed using the UMAT at Haukeland University Hospital, Bergen, Norway. ${ }^{13}$ In the present paper, we investigated 94 patients diagnosed with FD (80\% women, age $31.6 \pm 13.0$ years, body mass index [BMI] $\left.22.1 \pm 4.1 \mathrm{~kg} / \mathrm{m}^{2}\right), 88$ with IBS $(72 \%$ women, age $37.0 \pm$ 15.0 years, BMI $\left.23.8 \pm 4.6 \mathrm{~kg} / \mathrm{m}^{2}\right)$, and 66 patients with comorbid IBS and FD (from here on referred to as IBS + FD, 86\% women, age $37.6 \pm 13.0$ years, BMI $23.0 \pm 3.9 \mathrm{~kg} / \mathrm{m}^{2}$ ). For comparison, $30 \mathrm{HC}$ were recruited (66\% women, age $32.6 \pm 12.0$ years, BMI $23.5 \pm 2.6 \mathrm{~kg} / \mathrm{m}^{2}$ ). For the statistical analyses, we treated the patients with IBS + FD as a separate group. Patients with FD were generally younger $(P=0.011)$ and had a lower BMI $(P=0.047)$ than patients with IBS, but the difference in BMI disappeared when adjusting for postprandial nausea (logistic regression, adjusted $P=0.173$ ). We observed a larger age span in the IBS group. The gender distribution was not different between the groups $(P>$ 0.05). Results from some questionnaires are listed in Table 1. We found that IBS patients had higher scores on both IBS symptom severity scale (IBS-SSS) and GI specific anxiety (VSI) compared to patients suffering from $\mathrm{FD}(P<0.05)$. We observed no significant difference between the groups regarding neuroticism score (EPQ$\mathrm{N})$. All patient groups had significantly higher neuroticism scores than $\mathrm{HC}(P<0.001)$.

\section{Dyspeptic Symptoms in Irritable Bowel Syndrome and Functional Dyspepsia Compared to Healthy Controls}

Patients with FD, IBS or both had significantly more epigastric pain, nausea, and upper GI discomfort both before and after the soup meal $(P<0.001)$ compared to HC. The levels of satiety immediately after the meal was equal in all groups. Patients with IBS + FD reported higher levels of bloating/distention (mean VAS, 57.5; SD, 29.2) compared to HC (mean VAS, 40.2; SD, 25.4; $P$ $=0.006$ ) and patients with only IBS (mean VAS, 36.8; SD, 28.2; $P<0.001)$. In the fasting state, all patient groups reported higher levels of bloating/distention compared to $\mathrm{HC}(P<0.01)$. Not all patients were able to completely finish the soup meal of $500 \mathrm{~mL}$. Seventeen FD patients (18\%), 13 IBS + FD patients (20\%), and 5 IBS patients $(6 \%)$ were unable to finish, or vomited immediately after soup intake $(P=0.010)$. In the $\mathrm{HC}$ group, all participants 


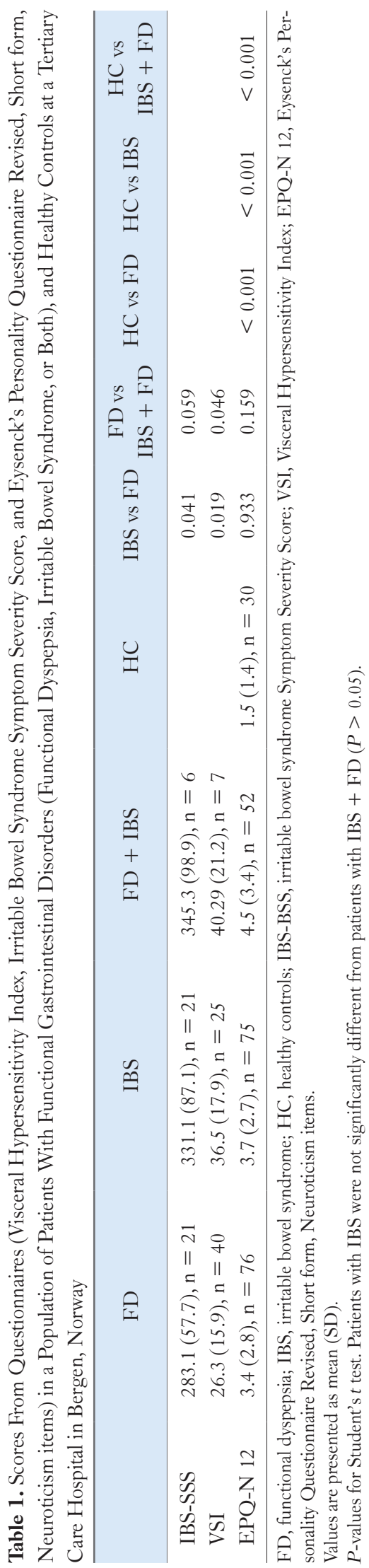

completed the meal. Not being able to complete the meal was associated with nausea (OR, 1.025; 95\% CI, 1.012-1.038; $P<0.001$ ), epigastric pain (OR, 1.017; 95\% CI, 1.004-1.030; $P=0.012)$, and upper GI discomfort (OR, 1.021; 95\% CI, 1.008-1.034; $P=$ $0.001)$ immediately after soup intake. Women were more likely to not complete the meal (OR, 5.14; 95\% CI, 1.204-22.400; $P=$

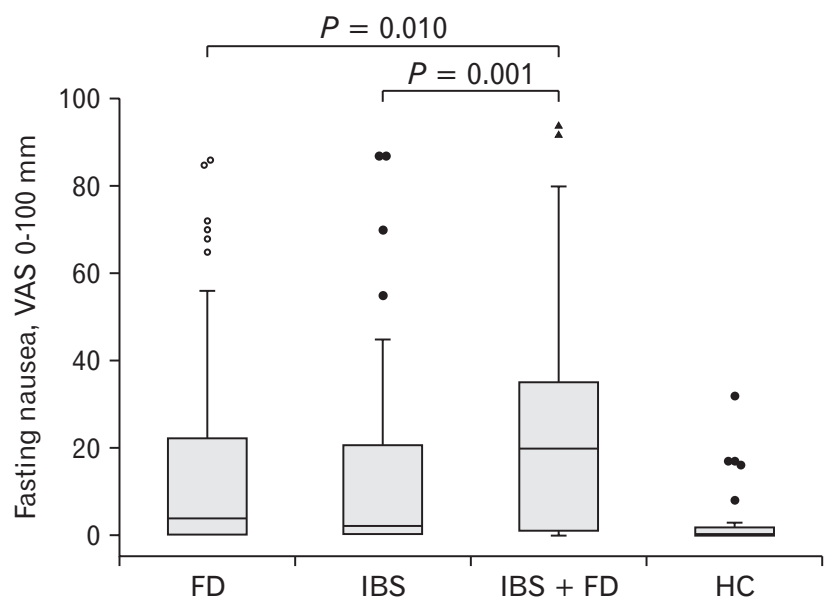

Figure 1. Fasting nausea. Self-reported nausea (visual analogue scale [VAS], 0-100 $\mathrm{mm}$ ) in a fasting state in patients with either functional dyspepsia (FD; $n=94)$, irritable bowel syndrome (IBS; $n=85)$, or both IBS and FD (IBS + FD; $\mathrm{n}=66$ ), compared to healthy controls $(\mathrm{HC} ; \mathrm{n}=30)$. All patient groups reported significantly higher levels of nausea compared to $\mathrm{HC}(P<0.005)$.

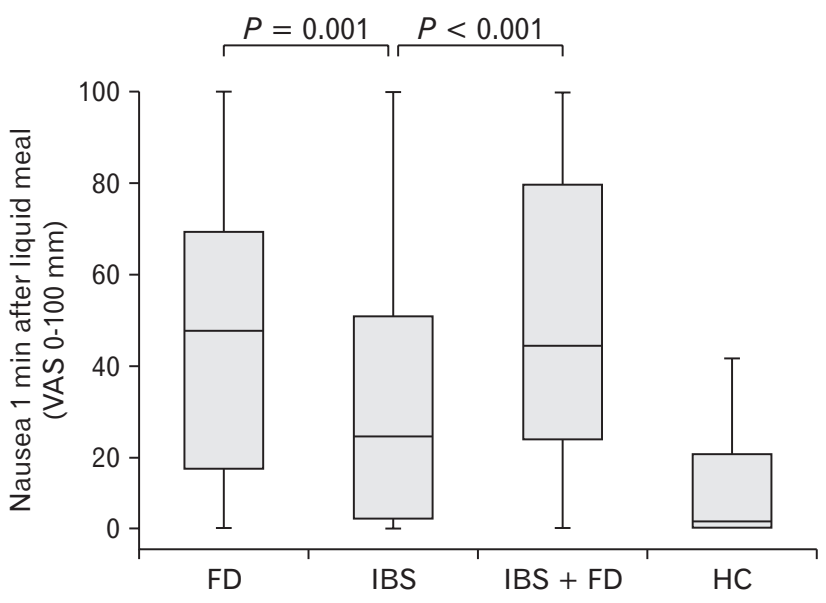

Figure 2. Postprandial nausea. Self-reported nausea (visual analogue scale [VAS], 0-100 mm) immediately after intake of $500 \mathrm{~mL}$ of a low calorie soup in patients with either functional dyspepsia (FD; $\mathrm{n}=$ 94), irritable bowel syndrome (IBS; $n=85$ ), or both IBS and FD $($ IBS $+\mathrm{FD} ; \mathrm{n}=66)$, compared to healthy controls $(\mathrm{HC} ; \mathrm{n}=30)$. All patient groups reported significantly higher levels of nausea compared to $\mathrm{HC}(P<0.001)$. 
0.015), and FD patients had higher probability of not finishing the meal compared to patients with IBS (OR, 3.665; 95\% CI, 1.29$10.41 ; P=0.010)$.

\section{Nausea in Irritable Bowel Syndrome and Functional Dyspepsia}

Patients with IBS, FD or both all reported higher levels of nausea in both fasting and postprandial condition (Fig. 1 and 2) compared to $\mathrm{HC}(P<0.001)$. BMI negatively correlated to nausea in both a fasting $(r=-0.202, P=0.010$, $)$ and a postprandial $(r=$ $-0.265, P=0.001)$ state. Furthermore, age negatively correlated to postprandial nausea $(r=-0.271, P<0.001)$. As shown in Table 2 , high proportions of patients in all patient groups reported nausea pre- and post-prandially, whereas only $3 \%$ of the healthy participants experienced nausea $>20 \mathrm{~mm}$ in a fasting state, and none of them reported nausea $>50 \mathrm{~mm}$ on VAS after the meal $(P<0.05)$. Almost half of the patients with FD reported symptoms of nausea of $>50 \mathrm{~mm}$ after the meal, and $25 \%$ of patients with IBS reported the same symptomes. The group with overlapping IBS and FD had the highest proportion of patients with nausea $>20 \mathrm{~mm}$ in a fasting state (52\%), compared to $26 \%$ (IBS) and $28 \%$ (FD), $P<$ 0.005 .

There was a correlation between the oblique fundal diameter at 20 minutes and nausea immediately after the meal $(r=0.196, P=$ 0.010 Pearson's correlation, all patients with IBS and/or FD, $\mathrm{n}=$ 202), but nausea did not correlate to neuroticism or GI specific anxiety score.

\section{Gastric Motility Assessed With Ultrasound}

Table 3 shows an overview of the gastric measurements from the UMAT in patients with IBS, FD, and HC, and Table 4 shows the results of the post-hoc analysis. The antrum was significantly larger compared to $\mathrm{HC}$ in a fasting state for FD patients $(P=$ $0.049)$, IBS patients $(P=0.006)$ and overlap FD + IBS patients $(P=0.010)$ (Fig. 3). Patients with FD had significantly smaller fundal diameters $(P<0.05$; Fig. 4$)$ and proximal areas $(P<0.05)$ after the liquid meal at 1 minute and 10 minutes postprandially compared to IBS patients. Compared to $\mathrm{HC}$, the sagittal area and oblique fundal diameter were smaller in patients with FD, but not the other patient groups, at 1 minute and 10 minutes (proximal area: $P<0.05$, and proximal diameter: $P<0.01$ ). IBS patients and the overlap group had similar proximal measurements as the HC group.

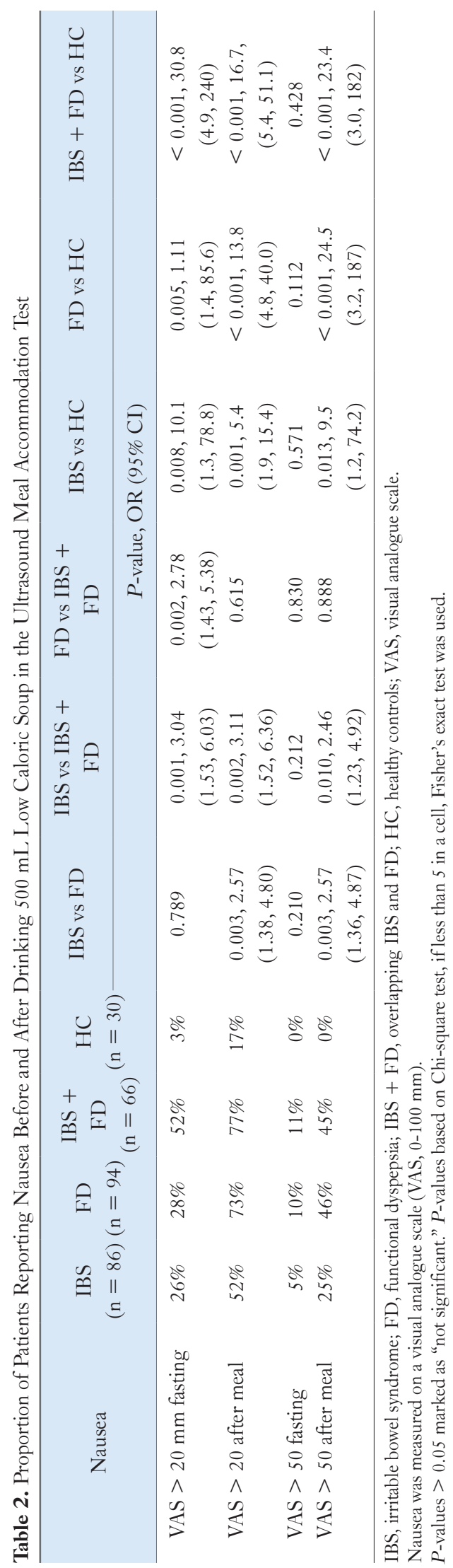


Table 3. Results From the Ultrasound Meal Accommodation Test

\begin{tabular}{|c|c|c|c|c|c|}
\hline Ultrasound measurement & $\mathrm{FD}(\mathrm{n}=75)$ & $\operatorname{IBS}(\mathrm{n}=80)$ & $\mathrm{FD}+\operatorname{IBS}(\mathrm{n}=51)$ & $\mathrm{HC}(\mathrm{n}=30)$ & $P$-value \\
\hline AA fasting & $4.0(1.9)$ & $4.1(1.6)$ & $4.4(2.4)$ & $3.2(1.1)$ & 0.031 \\
\hline $\mathrm{AA} 1 \mathrm{~min}$ & $14.1(5.4)$ & $13.1(4.7)$ & $15.6(6.6)$ & $11.7(5.1)$ & 0.009 \\
\hline $\mathrm{AA} 10 \mathrm{~min}$ & $10.6(4.6)$ & $10.2(4.6)$ & $11.2(6.2)$ & $8.8(4.3)$ & 0.197 \\
\hline AA $20 \mathrm{~min}$ & $8.4(4.8)$ & $7.5(4.0)$ & $8.1(4.4)$ & $6.7(3.8)$ & 0.292 \\
\hline $\mathrm{SA} 1 \mathrm{~min}$ & $26.4(6.2)$ & $29.1(5.7)$ & $27.3(5.6)$ & $29.4(4.2)$ & 0.012 \\
\hline $\mathrm{SA} 10 \mathrm{~min}$ & $22.3(6.0)$ & $25.5(5.6)$ & $22.9(5.4)$ & $24.9(3.5)$ & $0.002(\mathrm{~W})$ \\
\hline $\mathrm{SA} 20 \mathrm{~min}$ & $19.1(20.3)$ & $20.3(6.4)$ & $17.4(5.9)$ & $18.7(4.8)$ & 0.097 \\
\hline OFD $1 \mathrm{~min}$ & $5.5(2.0)$ & $6.5(1.8)$ & $6.2(1.4)$ & $6.7(1.0)$ & $0.001(\mathrm{~W})$ \\
\hline OFD $10 \mathrm{~min}$ & $4.5(1.8)$ & $5.4(1.6)$ & $5.1(1.4)$ & $5.6(1.0)$ & 0.004 \\
\hline OFD $20 \mathrm{~min}$ & $3.6(1.6)$ & $4.1(1.6)$ & $3.8(1.5)$ & $3.9(1.3)$ & 0.254 \\
\hline
\end{tabular}

FD, functional dyspepsia; IBS, irritable bowel syndrome; HC, healthy controls; AA, antral area; SA, sagittal area fundus; OFD, oblique frontal diameter fundus. Fasting and postprandial measurements of the distal and proximal stomach, in groups of patients with either FD or IBS, or overlapping FD and IBS (FD + IBS) compared to HC. Results from a 15-year retrospective study of patients referred to a tertiary care hospital in Norway.

One-way ANOVA results, in cases of different variance (tested by Levene's test), Welch's ANOVA was used, and marked as W. Post-hoc results (Games-Howell post-hoc test) are reported where ANOVA was significant.

Values are presented as mean (SD).

Table 4. Post-Hoc Analysis (Games-Howell Post-Hoc Test) of Ultrasound Measurements Where the One-way ANOVA Showed Significant Results

\begin{tabular}{|c|c|c|c|c|c|}
\hline \multirow[t]{2}{*}{$\begin{array}{l}\text { Ultrasound mea- } \\
\text { surement }\end{array}$} & $\begin{array}{l}\mathrm{FD} \text { vs } \\
\mathrm{HC}\end{array}$ & $\begin{array}{l}\text { FD vs } \\
\text { IBS }\end{array}$ & $\begin{array}{c}\text { FD vs } \\
\mathrm{FD}+ \\
\mathrm{IBS}\end{array}$ & $\begin{array}{c}\mathrm{FD}+ \\
\mathrm{IBS} \text { vs } \\
\mathrm{HC}\end{array}$ & $\begin{array}{c}\text { IBS vs } \\
\text { HC }\end{array}$ \\
\hline & \multicolumn{5}{|c|}{$P$-value } \\
\hline AA fasting & 0.049 & 0.963 & 0.635 & 0.010 & 0.006 \\
\hline $\mathrm{AA} 1 \mathrm{~min}$ & 0.152 & 0.577 & 0.524 & 0.020 & 0.573 \\
\hline $\mathrm{SA} 1 \mathrm{~min}$ & 0.031 & 0.026 & 0.826 & 0.267 & 0.994 \\
\hline $\mathrm{SA} 10 \mathrm{~min}$ & 0.031 & 0.004 & 0.957 & 0.216 & 0.920 \\
\hline OFD $1 \mathrm{~min}$ & $<0.001$ & 0.007 & 0.027 & 0.578 & 0.810 \\
\hline OFD $10 \mathrm{~min}$ & 0.003 & 0.022 & 0.137 & 0.576 & 0.856 \\
\hline
\end{tabular}

FD, functional dyspepsia; HC, healthy controls; IBS, irritable bowel syndrome; AA, antral area; SA, sagittal area fundus; OFD, oblique frontal diameter fundus.

Results from the Ultrasound Meal Accommodation Test. Fasting and postprandial measurements of the distal and proximal stomach, in groups of patients with either FD or IBS, or overlapping FD and IBS (FD + IBS) compared to HC. Results from a 15-year retrospective study of patients referred to a tertiary care hospital in Norway.

\section{Cut-off Values the Ultrasound Meal Accommodation Test}

To achieve a cut-off value for proximal accommodation, we calculated mean value $-2 \mathrm{SD}$ in $\mathrm{HC}$. Thus, an oblique frontal diameter 1 minute after soup $<4.7 \mathrm{~cm}$, and 10 minutes after soup $<3.5 \mathrm{~cm}$, and sagittal area $<20.9 \mathrm{~cm}^{2}$ at 1 minute and $<17.9 \mathrm{~cm}^{2}$ at $10 \mathrm{~min}-$ utes are indicative of impaired gastric accommodation. We found that $34 \%$ and $32 \%$ of patients with FD had oblique frontal diam-

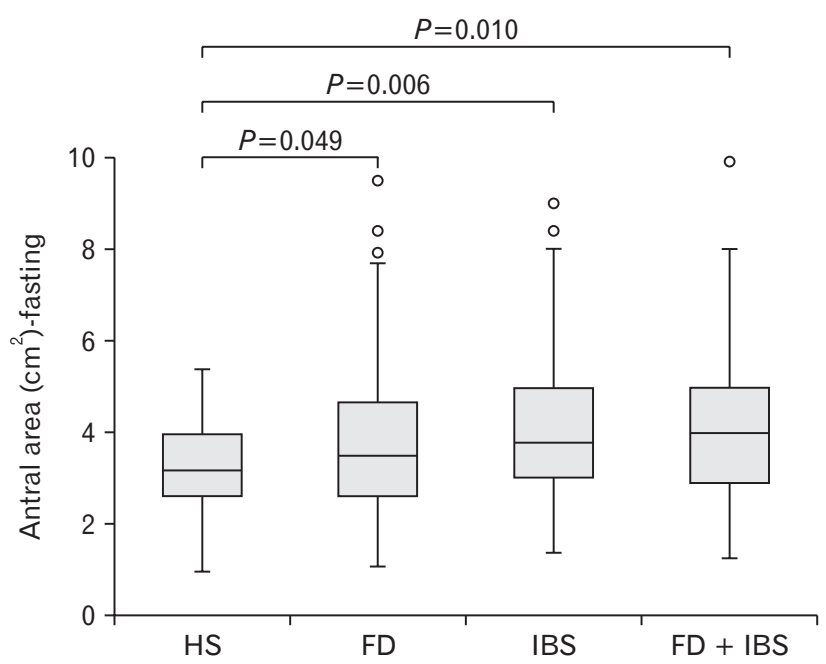

Figure 3. Antral area. Antral area in a fasting state measured with ultrasound in a sagittal section in patients with irritable bowel syndrome (IBS; $\mathrm{n}=80$ ), functional dyspepsia (FD; $\mathrm{n}=75$ ) or both IBS and FD (IBS + FD; $\mathrm{n}=51)$, compared to healthy controls ( HC; $\mathrm{n}=$ 30). All patient groups had larger antral areas in a fasting state compared to HC. VAS, visual analogue scale.

eters below the cut-off values at 1 minute and 10 minutes postprandially (compared to zero HC, $P<0.001$ ), but only $15 \%$ and $16 \%$ had proximal areas below the cut-off values (compared to $3 \% \mathrm{HC}$, $P>0.1)$. For antral distention, the cut-off values were calculated to be $>5.4 \mathrm{~cm}^{2}$ (fasting), $>21.9 \mathrm{~cm}^{2}$ (1 minute), $<17.3 \mathrm{~cm}^{2}$ (10 minutes), and $<14.2 \mathrm{~cm}^{2}$. A higher proportion of patients with $\mathrm{FD}$ had antral distention compared to $\mathrm{HC}$, but the difference was only significant in a fasting state $(\mathrm{FD}=16 \%$ vs $\mathrm{HC}=0 \%, P=0.033)$. 


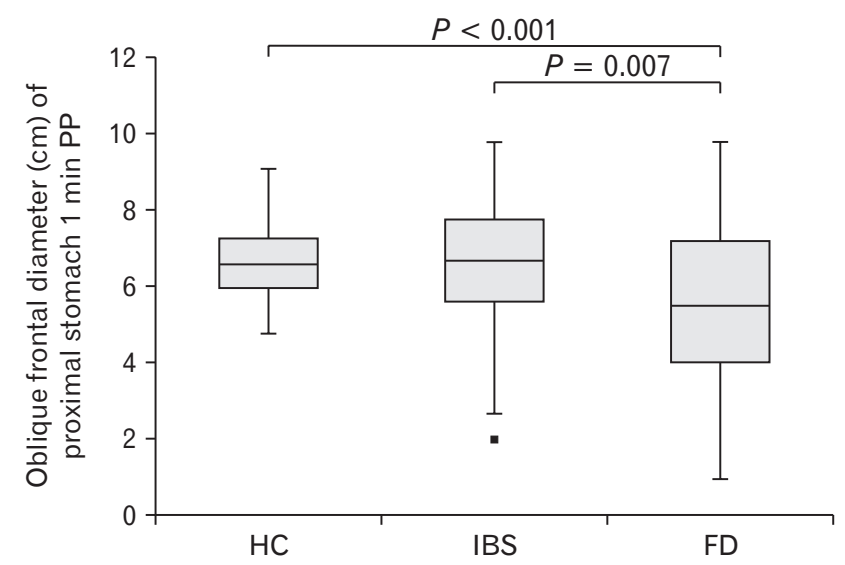

Figure 4. Oblique frontal diameter of the proximal stomach 1 minute postprandially (PP). Proximal diameter of the stomach measured in an oblique frontal section with ultrasound 1 minute after drinking a liquid, low-calorie soup, in patients with irritable bowel syndrome (IBS; $\mathrm{n}=80$ ), functional dyspepsia ( $\mathrm{FD} ; \mathrm{n}=75$ ) compared to healthy controls $(\mathrm{HC} ; \mathrm{n}=30)$. Patients with FD had smaller diameter of the proximal stomach compared to IBS and HC, indicating impaired accommodation.

\section{Neuroticism and Gastrointestinal Specific Anxiety and Its Relation to Dyspeptic Symptoms in Patients With Functional Dyspepsia and/or Irritable Bowel Syndrome}

Mean EPQ-N-scores for patients with FD and IBS were 3.4 and 3.7, respectively $(P>0.05$, not significant). In our HC group mean EPQ-N was $1.5(P<0.001)$. We found correlations between the following dyspeptic symptoms in a fasting state and EPQ-N: bloating/distention ( $r=0.215, P=0.002$; Fig. 5), upper abdominal discomfort $(r=0.171, P=0.015)$, and epigastric pain $(r=0.159, P=0.024)$. GI specific anxiety (VSI; $\mathrm{n}=58)$ correlated to epigastric pain $(r=0.308, P=0.008)$, bloating/distention $(r=0.255, P=0.030)$, and upper abdominal discomfort $(r=0.239, P=0.044)$ in a fasting state, but not postprandially. We found no correlations between VSI or EPQ-N and the gastric measurements.

\section{Discussion}

Symptoms from the upper GI tract as well as gastric motility has been studied in FD, but has been less investigated in IBS. In this study, we found that patients with IBS also experienced more symptoms of nausea, discomfort, and epigastric pain, immediately after a liquid meal compared with healthy subjects $(P<0.001)$.

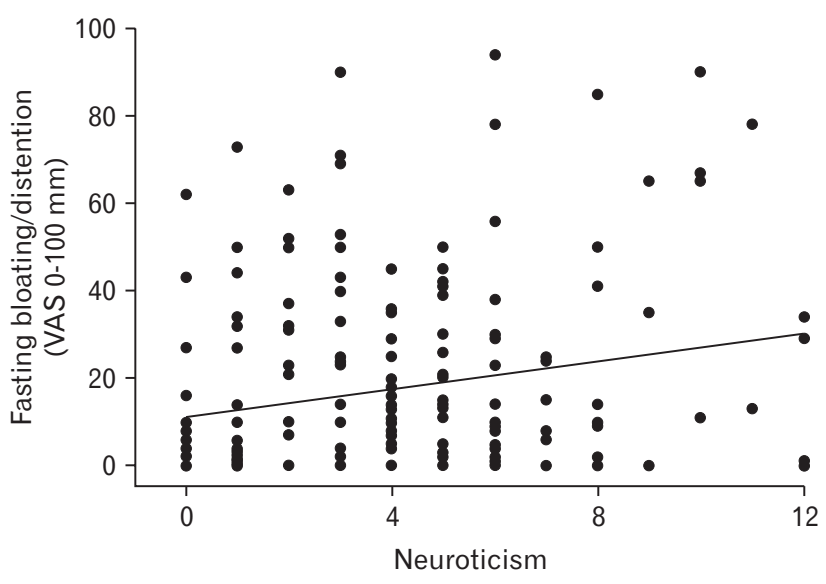

Figure 5. Bloating/distention and neuroticism. Scatter plot showing that neuroticism score (Eysenck's Personality Questionnaire Revised Short form, neuroticism items) was associated with symptoms of bloating/distention in a fasting state in patients with irritable bowel syndrome, functional dyspepsia or both $(\mathrm{n}=201 ; \mathrm{r}=0.215, P=$ 0.002). VAS, visual analogue scale.

In a fasting state, IBS patients reported higher levels of discomfort, nausea, bloating/distention, and epigastric pain than HC. Furthermore, we have for the first time shown that patients with IBS have larger antral area in a fasting state, equal to that of patients with $\mathrm{FD}^{20}{ }^{20}$ compared to $\mathrm{HC}(P=0.006)$.

In this study, we confirmed that the UMAT is a suitable method to assess gastric accommodation. Furthermore, we found that patients with IBS in general have a normal sized proximal stomach postprandially. We found a weak association between nausea and the oblique diameter of the proximal stomach 20 minutes after ingestion of the liquid meal. The size of the proximal stomach 20 minutes after meal ingestion is affected by both the accommodation reflex and subsequent adaptive relaxation, as well as the emptying rate of the stomach. Delayed gastric emptying is a well-known finding in some patients with $\mathrm{FD},{ }^{21}$ and it is reasonable to assume that a subgroup of the patients in our study had delayed emptying as well. However, we have not studied gastric emptying specifically in this protocol, and were not able to correct for this factor in our regression analyses. This may explain the weak correlations we observed. The patients in our material probably represents a very heterogenous population, with different underlying mechanisms behind their symptoms.

The relationship between proximal gastric accommodation and dyspeptic symptoms in FD is not fully elucidated. Some studies have found that impaired accommodation is correlated to symptoms of early satiety, postprandial fullness, or weight loss, and others have found no such correlation. ${ }^{21-23}$ Drinking capacity did not prove 
correlation to neither symptoms nor fundal accommodation, ${ }^{24}$ and some studies has found that the antral volume may be of greater importance for a patient's drinking capacity than proximal volume. ${ }^{25}$

Nausea is a non-specific symptom present in a variety of conditions, and is a frequently reported adverse effect of many drugs. It can be triggered by stimulation of the area postrema in the brainstem by medications, hormones, toxins or mechanical stimuli, ${ }^{26}$ or may be a consequence of diseases or dysfunctions throughout the GI system, relayed by the vagal nerve. ${ }^{27} \mathrm{~A}$ population survey showed that $9.5 \%$ of respondents had nausea at least once during the last 3 months. ${ }^{28}$ Although not a diagnostic criteria, nausea is a common complaint in patients with $\mathrm{FD}^{29}$ In adult populations, 39$65 \%$ of $\mathrm{FD}$ patients report nausea, ${ }^{30}$ and in a pediatric population studied in the United States, $86 \%$ of the patients reported nausea, of whom $70 \%$ reported an increase in nausea associated with eating. ${ }^{31}$ Nausea is frequently reported in IBS as well, ${ }^{2}$ and in one study $17 \%$ of IBS patients reported nausea during the last 7 days. ${ }^{32}$ However, nausea has not been the focus of many studies of patients with IBS and it is a component of FGID that is often difficult to treat adequately.

In this study we demonstrated that nausea can be induced by drinking a low-caloric meal in patients with FD as well as patients with IBS, but not in HC. We found that nausea was associated with lower BMI and young age. Symptoms arising within a few minutes after drinking suggests that the response is mainly due to gastric sensitivity to volume and stretching of the gastric wall, rather than hormonal or chemosensoric response. Thus, postprandial nausea may also be a sign of visceral hypersensitivity, similar to pain and discomfort. Interestingly, neuroticism- and VSI-scores did not correlate to self-reported nausea, neither fasting nor postprandially.

Symptoms of bloating/distention, upper abdominal discomfort, and epigastric pain correlated to neuroticism and GI specific anxiety in a fasting state. However, postprandial symptoms did not correlate to these psychological features. Hypersensitivity to stretch of the gastric wall is probably less affected by central processes, suggesting that local processes in the stomach are of great importance for generating postprandial dyspeptic symptoms. Other studies have similar findings, strengthening the hypothesis that visceral hypersensitivity is an important contributor to symptom generation in IBS and FD. ${ }^{33,34}$

The etiology of the FGIDs is complex. Whitehead et $\mathrm{al}^{35}$ suggested in 2002 a dual etiological mechanism for IBS, meaning that one group of IBS patients have predominantly central factors ("psychological"), whereas others have a peripheral ("biological”) mechanism. This theory was supported in a mixture model analysis by
Polster et $\mathrm{al}^{36}$ in 2017, showing that IBS patients could be separated into several subgroups based not only on their bowel habits, but on the presence of extraintestinal psychiatric or somatic symptoms. We observed that only a subset of our patients had high scores on questionnaires such as VSI and EPQ-N 12, supporting the view that psychological factors are of importance for some groups of FGID patients, but maybe not all.

Being a retrospective cross-sectional study, it has limited value concerning causality of the associations we observed. A disadvantage of the study is that the use of different questionnaires has varied over the years, accounting for missing data in some questionnaires.

As the high mean IBS-SSS score indicated, the patient population represents that of a specialized referral center rather than a general population of FGID patients. Accordingly, we should be careful to extrapolate our findings to the general population. However, the study is unique in combining results from a drinking test, ultrasound measurements of the stomach, and psychological questionnaires.

In conclusion, nausea is a common symptom in patients with IBS as well as in FD, and was associated with low BMI and age, but not correlated to anxiety and neuroticism. For the first time, we have shown that patients with IBS, as well as patients with FD, had enlarged antral areas in a fasting state. Patients with FD had lower measurements of the proximal stomach as a sign of impaired accommodation, while patients with IBS had normal accommodation. Symptoms of bloating/distention, epigastric pain, and upper abdominal discomfort all correlated to anxiety and neuroticism in a fasting state, but not postprandially. Accordingly, postprandial symptoms were not influenced by the individual's anxiety or neurotic personality traits. This study support the view that there is a great overlap between various FGIDs, and we have for the first time demonstrated that patients with IBS also exhibit fasting antral distention on ultrasound scanning.

Financial support: Elisabeth K Steinsvik has received funding from the Western Norway Regional Health Authority (Grant No. 912124).

\section{Conflicts of interest: None.}

Author contributions: Elisabeth K Steinsvik collected and systemized data from patient records, analyzed the data, and written the paper; Jørgen Valeur contributed with comments on the paper, and was active in the planning of the analyses; and Odd $\mathrm{H}$ Gilja and Trygve Hausken have conducted all the ultrasound examina- 
tions, and contributed to writing of the paper.

\section{References}

1. Vanheel H, Farré R. Changes in gastrointestinal tract function and structure in functional dyspepsia. Nat Rev Gastroenterol Hepatol 2013;10:142-149.

2. Mearin F, Lacy, Brian E, et al. Bowel disorders In: Drossman DA, ed. Rome IV functional gastrointestinal disorders, disorders of the gut-brain interaction. raleigh, NC: The rome foundation 2016:980.

3. Mearin F, Lacy BE, Chang L, et al. Bowel disorders. Gastroenterology 2016;150:1393-1407, e5.

4. Stanghellini V, Chan FK, Hasler WL, et al. Gastroduodenal disorders. Gastroenterology 2016;150:1380-1392.

5. Talley NJ, Ford AC. Functional dyspepsia. N Engl J Med 2015;373:1853-1863

6. Kim BJ, Kuo B. Gastroparesis and functional dyspepsia: a blurring distinction of pathophysiology and treatment. J Neurogastroenterol Motil 2019;25:27-35.

7. Enck P, Azpiroz F, Boeckxstaens G, et al. Functional dyspepsia. Nat Rev Dis Primers 2017;3:17081.

8. Chey WD. The role of food in the functional gastrointestinal disorders: introduction to a manuscript series. Am J Gastroenterol 2013;108:694697.

9. Mykletun A, Jacka F, Williams L, et al. Prevalence of mood and anxiety disorder in self reported irritable bowel syndrome (IBS). An epidemiological population based study of women. BMC Gastroenterol 2010;10:88.

10. Muscatello MR, Bruno A, Scimeca G, Pandolfo G, Zoccali RA. Role of negative affects in pathophysiology and clinical expression of irritable bowel syndrome. World J Gastroenterol 2014;20:7570-7586.

11. Tack J, Van den Houte K, Carbone F. Gastroduodenal motility disorders. Curr Opin Gastroenterol 2018;34:428-435.

12. Gilja OH, Hausken T, Odegaard S, Berstad A. Monitoring postprandial size of the proximal stomach by ultrasonography. J Ultrasound Med 1995;14:81-89.

13. Steinsvik EK, Hausken T, Gilja OH. The ultrasound meal accommodation test in 509 patients with functional gastrointestinal disorders. Scand J Gastroenterol 2016;51:778-794.

14. Hasler WL, Li BU, Koch KL, Parkman HP, Kovacic K, McCallum RW. Methodologic considerations for studies of chronic nausea and vomiting in adults and children. Auton Neurosci 2017;202:28-39.

15. Gilja OH, Lunding J, Hausken T, Gregersen H. Gastric accommodation assessed by ultrasonography. World J Gastroenterol 2006;12:28252829.

16. Eysenck SB, Eysenck HJ, Barrett P. A revised version of the psychoticism scale. Pers Individ Dif 1985;6:21-29.

17. Labus JS, Bolus R, Chang L, et al. The visceral sensitivity index: development and validation of a gastrointestinal symptom-specific anxiety scale. Aliment Pharmacol Ther 2004;20:89-97.

18. Labus JS, Mayer EA, Chang L, Bolus R, Naliboff BD. The central role of gastrointestinal-specific anxiety in irritable bowel syndrome: further validation of the visceral sensitivity index. Psychosom Med 2007;69:8998.

19. Francis CY, Morris J, Whorwell PJ. The irritable bowel severity scoring system: a simple method of monitoring irritable bowel syndrome and its progress. Aliment Pharmacol Ther 1997;11:395-402.

20. Hausken T, Svebak S, Wilhelmsen I, et al. Low vagal tone and antral dysmotility in patients with functional dyspepsia. Psychosom Med 1993;55:12-22.

21. Vanheel H, Carbone F, Valvekens L, et al. Pathophysiological abnormalities in functional dyspepsia subgroups according to the rome III criteria. Am J Gastroenterol 2017;112:132-140.

22. Van Oudenhove L, Vandenberghe J, Geeraerts B, et al. Determinants of symptoms in functional dyspepsia: gastric sensorimotor function, psychosocial factors or somatisation? Gut 2008;57:1666-1673.

23. Boeckxstaens GE, Hirsch DP, Kuiken SD, Heisterkamp SH, Tytgat GN. The proximal stomach and postprandial symptoms in functional dyspeptics. Am J Gastroenterol 2002;97:40-48.

24. Boeckxstaens GE, Hirsch DP, van den Elzen BD, Heisterkamp SH, Tytgat GN. Impaired drinking capacity in patients with functional dyspepsia: relationship with proximal stomach function. Gastroenterology 2001;121:1054-1063.

25. Sarnelli G, Caenepeel P, Geypens B, Janssens J, Tack J. Symptoms associated with impaired gastric emptying of solids and liquids in functional dyspepsia. Am J Gastroenterol 2003;98:783-788.

26. Miller AD, Leslie RA. The area postrema and vomiting. Front Neuroendocrinol 1994;15:301-320.

27. Koch KL. Physiology of nausea. In: Koch KL, Hasler WL, eds. Nausea and vomiting: diagnosis and treatment. Cham: Springer international publishing 2017:1-13.

28. Camilleri M, Dubois D, Coulie B, et al. Prevalence and socioeconomic impact of upper gastrointestinal disorders in the United States: results of the US upper gastrointestinal study. Clin Gastroenterol Hepatol 2005;3:543-552.

29. Harer KN, Pasricha PJ. Chronic unexplained nausea and vomiting or gastric neuromuscular dysfunction (GND)? An update on nomenclature, pathophysiology and treatment, and relationship to gastroparesis. Curr Treat Options Gastroenterol 2016;14:410-419.

30. Tack J, Talley NJ. Functional dyspepsia--symptoms, definitions and validity of the Rome III criteria. Nat Rev Gastroenterol Hepatol 2013;10:134141.

31. Friesen CA, Rosen JM, Schurman JV. Prevalence of overlap syndromes and symptoms in pediatric functional dyspepsia. BMC Gastroenterol 2016;16:75.

32. Shah ED, Almario CV, Spiegel BMR, Chey WD. Lower and upper gastrointestinal symptoms differ between individuals with irritable bowel syndrome with constipation or chronic idiopathic constipation. J Neurogastroenterol Motil 2018;24:299-306.

33. Elsenbruch S, Rosenberger C, Enck P, Forsting M, Schedlowski M, Gizewski ER. Affective disturbances modulate the neural processing of visceral pain stimuli in irritable bowel syndrome: an fMRI study. Gut 2010;59:489-495. 
34. Simrén M, Törnblom H, Palsson OS, et al. Visceral hypersensitivity is associated with GI symptom severity in functional GI disorders: consistent findings from five different patient cohorts. Gut 2018;67:255-262.

35. Whitehead WE, Palsson O, Jones KR. Systematic review of the comorbidity of irritable bowel syndrome with other disorders: what are the causes and implications? Gastroenterology 2002;122:1140-1156.
36. Polster A, Van Oudenhove L, Jones M, ÖHman L, Törnblom H, Simrén M. Mixture model analysis identifies irritable bowel syndrome subgroups characterised by specific profiles of gastrointestinal, extraintestinal somatic and psychological symptoms. Aliment Pharmacol Ther 2017;46:529-539. 